\title{
Epithelial markers in prostatic, bladder, and colorectal cancer: an immunoperoxidase study of epithelial membrane antigen, carcinoembryonic antigen, and prostatic acid phosphatase
}

\author{
EADIE HEYDERMAN, BRENDA ME BROWN, TC RICHARDSON* \\ From the Department of Histopathology, St Thomas's Hospital, London SE1 7EH
}

SUMMARY Twenty prostatic adenocarcinomas, 20 transitional cell carcinomas of the bladder, and 20 colorectal adenocarcinomas were stained for epithelial membrane antigen, carcinoembryonic antigen, and prostatic acid phosphatase. Polyclonal affinity purified first and second antibodies and an indirect immunoperoxidase technique were used. All of the colorectal and bladder tumours and 16/20 prostatic tumours were positive for epithelial membrane antigen. All 20 colorectal, 7/20 bladder, and 5/20 prostatic tumours stained for carcinoembryonic antigen. All of the prostatic adenocarcinomas and none of the colorectal or bladder tumours were positive for prostatic acid phosphatase. These markers may be used to discriminate between tumours arising from these sites.

The histopathological differential diagnosis of widely invasive tumours of prostate, rectum, and bladder may, on occasion, be difficult on morphological grounds alone, especially when the tumours appear undifferentiated on a haematoxylin and eosin preparation. Even though the tumour shows evidence of adenocarcinomatous differentiation, there may be clinical doubt as to which of these organs is the primary source. Furthermore, tumours from these sites may present with nodal metastases or with a pathological fracture, with no definite signs or symptoms to indicate the likely primary site. It is important to make the correct diagnosis, since clinical management and treatment, even if only palliative, is entirely different for these three tumours.

This study was designed to investigate the distribution of epithelial membrane antigen (EMA), carcinoembryonic antigen (CEA), ${ }^{2}$ and prostatic acid phosphatase in tissue sections ${ }^{3-6}$ to see whether their expression was sufficiently different to be of value in histological diagnosis.

Accepted for publication 9 August 1984

*Present address: MRC Radiobiology Unit, Harwell, Didcot, Oxford.
EMA $^{1}$ is a large glycoprotein, $>440000$ daltons, which has now been partially purified.' It is recognised by antisera raised against milk fat globule membranes, ${ }^{8}$ and has been shown in the prostate, ${ }^{1910}$ bladder, ${ }^{9}$ and colon, ${ }^{19}$ as well as in a wide variety of other benign and malignant lesions. ${ }^{19}$

CEA is an incompletely defined glycoprotein of 180000 daltons. It was originally found in colorectal carcinomas, ${ }^{2}$ and has since been shown by immunocytochemical techniques in a number of other sites including the prostate ${ }^{10-13}$ and bladder. ${ }^{12} 14-17$

Prostatic acid phosphatase is an isoenzyme of acid phosphatase (ortho phosphoric monoester phosphohydrolase, EC $3 \cdot 1 \cdot 3 \cdot 2$ ) of 100000 daltons $^{18}$ and isoelectric point $4 \cdot 2-5 \cdot 5$. Its enzymic activity is largely destroyed by formalin fixation and processing of tissue into paraffin wax, but specific antisera may be used to demonstrate prostatic acid phosphatase in sections of fixed prostatic tumours and of their metastases, as well as in benign prostatic tissue.$^{4-6}$ Cross reactivity with pancreatic islet cells, salivary glands, renal carcinomas and breast carcinomas with some rabbit antisera has been reported, ${ }^{19-22}$ although no such activity has been seen in 142 various non-prostatic benign and malignant lesions stained with the affinity purified antibody used in this study (unpublished data). 


\section{Material and methods}

Formalin fixed, paraffin embedded blocks of 20 adenocarcinomas of the prostate, 20 transitional cell carcinomas of the bladder, and 20 colorectal adenocarcinomas were selected from the routine surgical files (Table 1). In this study only one block of each tissue was used, as previous experiments had indicated that where these particular markers were present all of the blocks were likely to be positive and often only one block was available from bladder biopsies. Where possible, normal tissue was included to act as an inbuilt control.

Sections were cut at $4 \mu \mathrm{m}$ on to slides coated with chrome gelatin to prevent the sections floating during immunostaining. An indirect immunoperoxidase technique was used as described previously, ${ }^{23}$ and endogenous peroxidase was inhibited by a sequence of $6 \%$ hydrogen peroxide, $2.5 \%$ periodic acid, and then $0.02 \%$ potassium borohydride. ${ }^{24}$ Indirect peroxidase conjugates were prepared by periodate oxidation $^{25}$ using affinity purified goat antirabbit immunoglobulin.

Table 1 Differentiation in colorectal, bladder, and prostatic tumours*

\begin{tabular}{llll}
\hline & Well & Moderately & Poorly \\
\hline Ca colon & 1 & 18 & 1 \\
Ca bladder & 8 & 8 & 4 \\
Ca prostate & 6 & 11 & 3 \\
\hline
\end{tabular}

*This distribution of differentiation in 20 of each type of tumour reflects that seen in tumours chosen at random from those reported during 1981-3.

\section{ANTISERA AND CONTROLS}

The primary antisera were all raised in rabbits and affinity purified on agarose columns (AffiGel 10, BioRad Laboratories Ltd, Herts), to which the relevant antigen had been bound. Specificity was demonstrated by repassage of the affinity purified antibody down the column. Staining could be shown in the appropriate positive control tissue with the eluate from this second passage and was absent from the (unbound) effluent. This latter effluent was used as a negative control. ${ }^{26}$

Milk fat globule membranes were prepared by freeze-thawing an aqueous suspension of the washed cream fraction of whole human milk ${ }^{8}$ and pelleted by high speed centrifugation. CEA had been prepared from a hepatic metastasis of a colorectal carcinoma and was a generous gift from Dr Westwood (ICRF, Sutton). Prostatic acid phosphatase was isolated from pooled seminal plasma obtained from the vasectomy clinic. The enzyme was precipitated with ammonium sulphate and further purified by column chromatography, first by ion exchange on a DEAE AffiGel blue column (Biorad Laboratories Ltd, Herts) and then by gel filtration on a Sephadex G200 column (Pharmacia (GB) Ltd, Bucks). ${ }^{27} 28$

The immunogens were emulsified in a nonulcerogenic adjuvant (Guildhay, University of Surrey) and injected subcutaneously into New Zealand rabbits.

\section{CONTROLS}

The positive control for CEA was a colonic carcinoma; for EMA a moderately differentiated infiltrating ductal carcinoma of the breast was chosen; and for prostatic acid phosphatase a prostate showing benign adenomyomatous hyperplasia was used. Each positive section of positive control or test was matched with a control section treated with the negative "absorbed" antibody.

\section{Results (Table 2)}

All of the colorectal and bladder carcinomas and $16 / 20$ prostatic carcinomas were positive for EMA (Figs. 1 and 2). All 20 colorectal, 7/20 bladder, and $5 / 20$ prostatic carcinomas were positive for CEA (Figs. 3 and 4). All of the prostatic tumours (Figs. 5 and 6) and none of the bladder or colorectal care cinomas were positive for prostatic acid phosphat ase.

Table 2 Results of immunoperoxidase stains for epithelial membrane antigen (EMA), carcinoembryonic antigen $(C E A)$, and prostatic acid phosphatase (PAP)

\begin{tabular}{lccc}
\hline & $E M A$ & $C E A$ & $P A P$ \\
\hline Colorectal & $20 / 20$ & $20 / 20$ & $0 / 20$ \\
Bladder & $20 / 20$ & $7 / 20$ & $0 / 20$ \\
Prostate & $16 / 20$ & $5 / 20$ & $20 / 20$ \\
\hline
\end{tabular}

Without prior knowledge, it was not possible to tell which antibody had been used because the pattern of staining was so similar for the three epithelial markers, although stains for EMA in colorectal tumours were weaker than those for CEA, and stains for CEA in prostatic carcinomas (Fig. 4) were weaker than in colorectal tumours (Fig. 3). The dilution of primary antibody had been determined on the appropriate positive control tissue-breast carcinoma for EMA, colorectal carcinoma for CEA, and benign prostatic hypertrophy for prostatic acid phosphatase. These differences were therefore relative and could be assessed only in relation to those tissues.

CARCINOEMBRYONIC ANTIGEN

There was no correlation between the presence or 

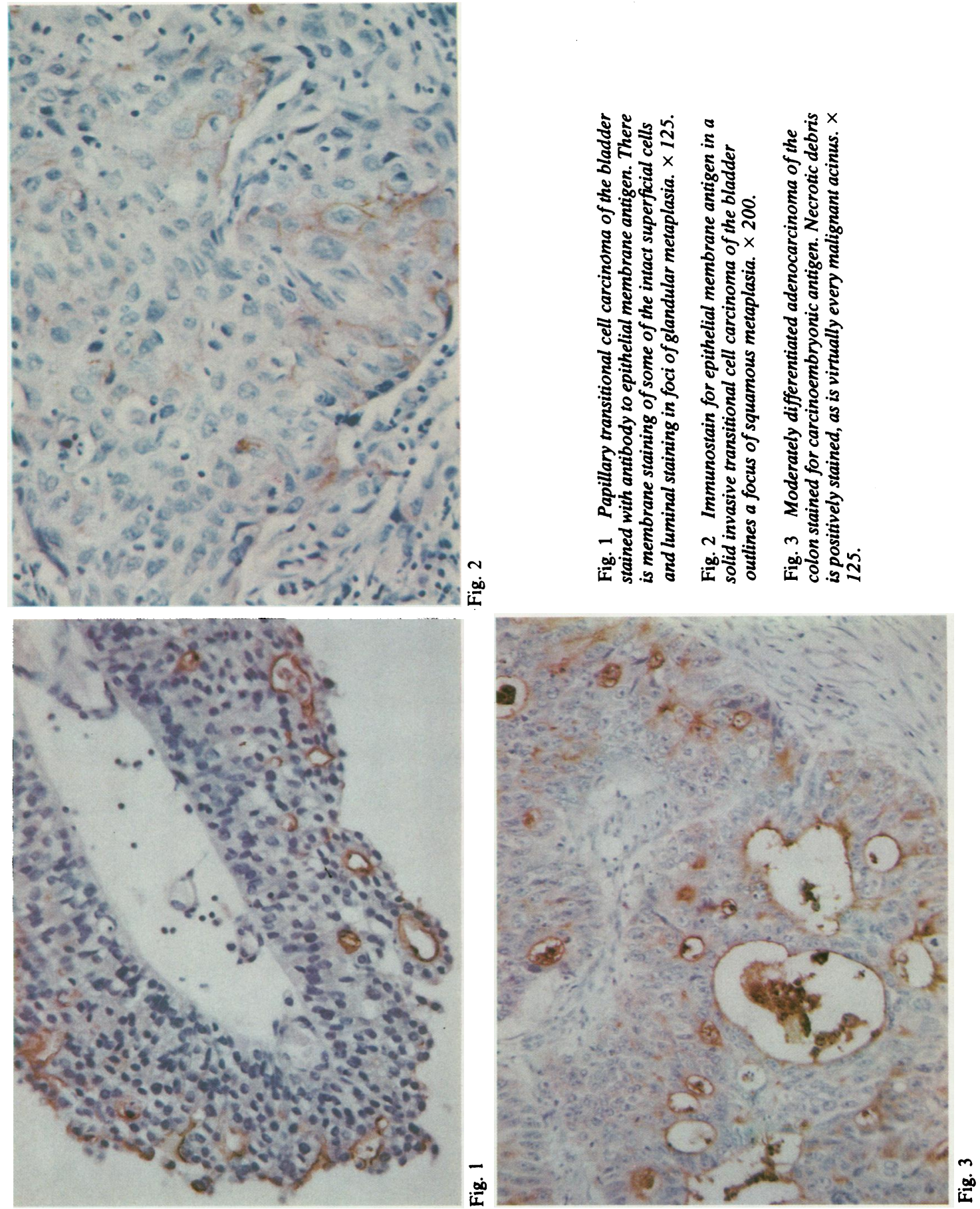

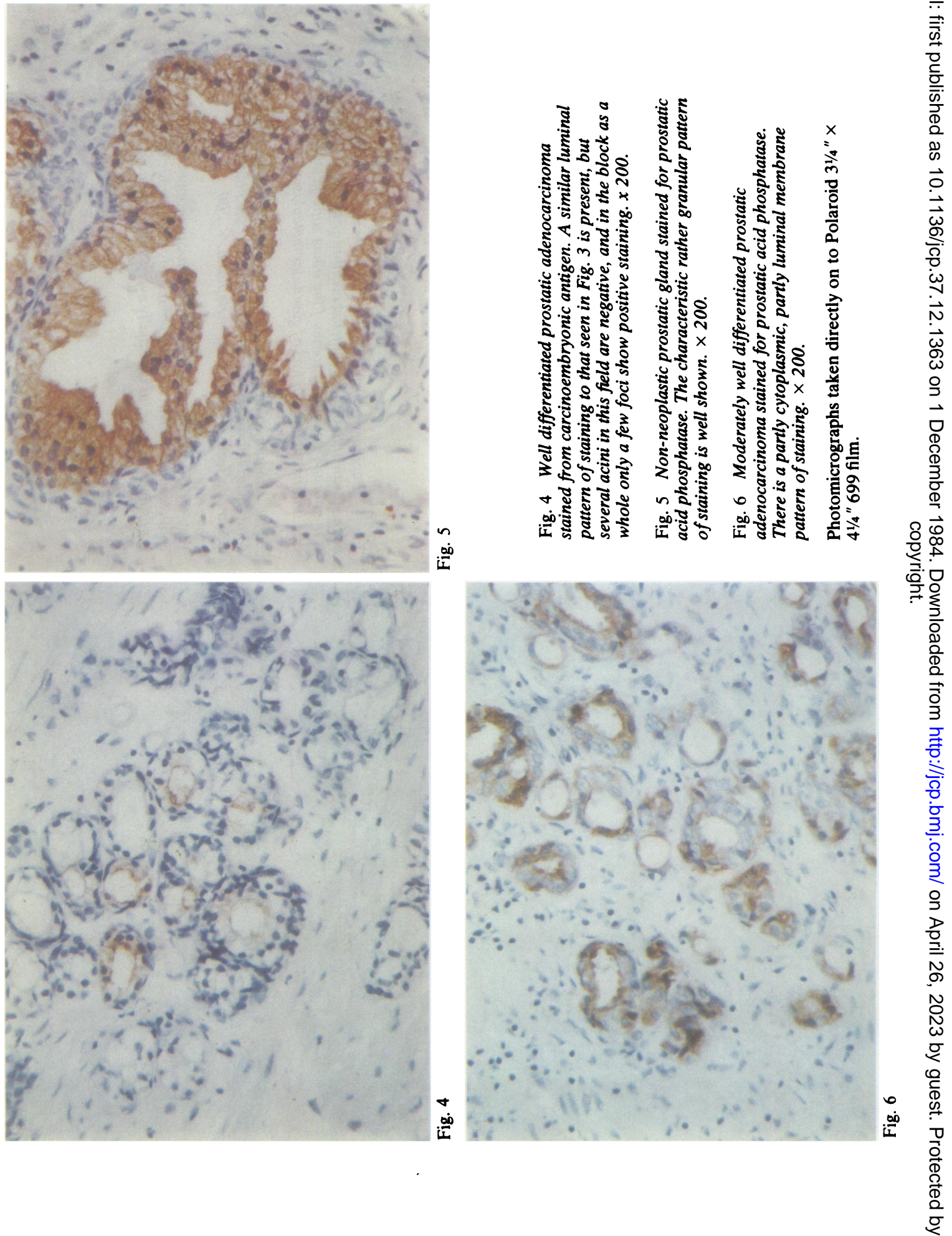
absence of CEA or EMA and degree of differentiation or morphology in the prostatic tumours. Where present, positivity for CEA was found mainly on the luminal membrane in well or moderately differentiated colorectal or prostatic adenocarcinomas and in the cytoplasm of poorly differentiated tumours. In sections of bladder tumours, CEA was largely confined to those areas showing squamous metaplasia and was also found on non-malignant transitional cell epithelium undergoing squamous transformation. In the prostate, CEA was mainly present on luminal membranes, but was always weaker than in colorectal carcinomas (Figs. 3 and 4).

\section{EPITHELIAL MEMBRANE ANTIGEN}

Stains for EMA were present on the cell membranes of transitional cell carcinomas and outlined foci of glandular (Fig. 1) and squamous differentiation (Fig. 2). It was interesting that many more transitional cell carcinomas contained foci of glandular metaplasia shown by the EMA stain than was apparent with haematoxylin and eosin or periodic acid Schiff preparations. In colorectal and prostatic adenocarcinomas both CEA and EMA were present mainly on the luminal membranes of malignant acini, with some cytoplasmic staining particularly in poorly differentiated carcinomas.

PROSTATIC ACID PHOSPHATASE

Positive staining for prostatic acid phosphatase was found in non-neoplastic prostatic glands (Fig. 5) and in $20 / 20$ prostatic tumours (Fig. 6), although not all benign or malignant glands in any one section were positive. It was found mainly in the cytoplasm, with some luminal membrane staining. No positivity was found in non-prostatic tumours.

No staining was seen with the negative "absorbed" controls for any of the three markers.

\section{Discussion}

These results confirm previous reports that the demonstration of prostatic acid phosphatase in a tumour is virtually diagnostic of prostatic origin. A further 142 primary and metastatic tumours from a wide variety of sites outside the prostate have been stained with the antibody used here, and none has been positive (unpublished data). Although there were no false positives, the possibility of false negatives cannot be excluded, although in most studies between $93 \%$ and $100 \%$ of prostatic carcinomas have been found to be positive..$^{50102124-32}$ Shevchuk et $a^{3.3}$ found lower rates of positivity using polyclonal goat ( $83 \%$ positive), rabbit $(70 \%)$, or monoclonal antibodies (59\%).

Since this study was carried out, and the high degree of specificity of the antibody to prostatic acid phosphatase was demonstrated, the antibody has been shown to be particularly valuable in distinguishing between solid transitional cell carcinomas of the bladder and poorly differentiated prostatic carcinoma in cases verified at operation or necropsy. Prostatic ducts lined by transitional type epithelium show some positivity, however, and transitional cell carcinomas of prostatic ducts are currently being studied. Determination of the physical site of origin of tumours at the bladder neck may be difficult when prostatic chippings, rather than a prostatectomy specimen, are available.

Antibodies to a smaller protein, prostate specific antigen of 34000 daltons and isoelectic point $6 \cdot 9,{ }^{27}$ do not apparently show the cross reactivity with non-prostatic tissues reported with some antisera to prostatic acid phosphatase and show similar positivity rates in prostatic carcinoma. Antibodies to prostatic acid phosphatase have an advantage over anti-prostate specific antigen, however, in that the effects of various fixatives and processing schedules in terms of both antigenic and enzymic reactivity can be studied and the affinity purified antibody used in this study has not so far been found to stain nonprostatic tissues.

Eighty colorectal carcinomas have now been stained by us for CEA" (unpublished data). As found by others, ${ }^{12} 34$ all have been CEA positive, so that the absence of CEA makes the diagnosis of a colorectal primary tumour unlikely. Wiley et al, ${ }^{35}$ however, found only $21 / 41(51 \%)$ colonic tumours positive for CEA using an unpurified and unabsorbed rabbit antibody (source not stated).

Our finding of CEA in 7/20 (35\%) bladder carcinomas is comparable with that of Wahren, ${ }^{16}$ who examined cytological preparations with a positivity of $18 / 40(48 \%)$. Wiley et al ${ }^{17}$ found CEA in $26 / 48$ (58\%), but since they used a commercial rabbit antibody without mention of spleen absorption they may well have been showing the shared normal cross reacting antigen (NCA) determinant. ${ }^{3637}$ The number of CEA positive tissues reported by Goldenberg et al in $1976^{14}$ is generally lower than is presently found with more recently developed reagents: $23 / 37(62 \%)$ of their colorectal carcinomas, $1 / 10(10 \%)$ of bladder, and none of their 10 prostatic tumours were positive for CEA. In their 1978 paper $^{\text {s }}$ 60/71 (85\%) colorectal and 4/38 (11\%) bladder tumours were positive.

In the study reported here $5 / 20(25 \%)$ of the prostatic carcinomas were positive for CEA, a similar figure to the $7 / 30(23 \%)$ reported by Heidl. ${ }^{12}$ This is different from the figures of $16 / 38(42 \%)$ before absorption with spleen and 1/38 (2.6\%) after absorption reported by Purnell et al.$^{13}$ 
The main value of the EMA immunostain in these tumours lies in the distinction of carcinoma from lymphoma or sarcoma. ${ }^{1938}$ Fifty six of the 60 carcinomas in this study were positive. Sloane and Ormerod" found $2 / 2$ prostatic carcinomas, $4 / 4$ bladder tumours, and $6 / 6$ colorectal carcinomas were positive, and using a similar rabbit unpurified antibody Ellis $e$ al $^{10}$ found EMA in 47/60 (78\%).

Variations in the percentage of positive tumours found in published reports are probably largely due to the use of antisera from different sources, although the immunoperoxidase technique employed and differences in fixation and processing schedules are important. It is advisable for departments which intend to use immunocytochemical stains as an adjunct to diagnostic histopathology to carry out immunostains on a number of tissues fixed and processed at that institution. The incidence of positive tumours stained under local conditions may be ascertained and the potential value of the antibodies established.

We thank the staff of the Department of Histopathology at St Thomas's Hospital for sectioning and haematoxylin and eosin and periodic acid Schiff stains. CEA was kindly provided by $\mathrm{Dr}$ Westwood (ICR, Sutton) and goat antirabbit immunoglobulin by Messrs Hoffman La-Roche (New Jersey, USA). The work was supported in part by the Cancer Research Campaign and in part by St Thomas's Hospital Research Endowments Fund.

\section{References}

' Heyderman E, Steele K, Ormerod MG. A new antigen on the epithelial membrane: its immunoperoxidase localisation in normal and neoplastic tissue. J Clin Pathol 1979;32:35-9.

${ }^{2}$ Gold P, Freedman SO. Demonstration of tumour-specific antigens in human colonic carcinomata by immunological tolerance and absorption techniques. J Exp Med 1965; 121:439-62.

"Gutman AB, Gutman EB. An "acid" phosphatase occurring in the serum of patients with metastasizing carcinoma of the prostate gland. J Clin Invest 1938; 17:473-6.

${ }^{4}$ Burns J. Prostatic acid phosphatase in tissue sections revealed by the unlabelled antibody peroxidase-antiperoxidase method Biomedicine 1977; 27:7-10.

s Pontes JE, Choe B, Rose N, Pierce JM. Indirect immunofluorescence for identification of prostatic epithelial cells. J Urol 1977; 117:459-62.

- Jobsis AC, De Vries GP, Meijer AEFH, Ploem JS. The immunohistochemical detection of prostatic acid phosphatase: its possibilities and limitations in tumour histochemistry. Histochem J 1981;13:961-73.

' Ormerod MG, Steele K, Westwood JH, Mazzini MN. Epithelial membrane antigen: partial purification, assay and properties. Br J Cancer 1983; 48:533-41.

"Ceriani RL, Thompson K, Peterson JA, Abraham S. Surface differentiation antigens of human mammary epithelial cells carried on the human milk fat globule. Proc Nat Acad Sci USA 1977;74:582-6.

- Sloane JP, Ormerod MG. Distribution of epithelial membrane antigen in normal and neoplastic tissues and its value in diag- nostic tumor pathology. Cancer 1981;47:1786-95.

" Ellis DW, Leffers S, Davies JS, Ng ABP. Multiple immunoperoxidase markers in benign hyperplasia and adenocarcinoma of the prostate. Am J Clin Pathol 1984;81:279-84.

" Heyderman E, Neville AM. A shorter immunoperoxidase technique for the demonstration of carcinoembryonic antigen and other cell products. J Clin Pathol 1977;30.138-40.

12 Heidl G. Demonstration of carcinoembryonic antigen (CEA) by means of the immunoperoxidase technique in paraffinembedded specimens of tumour tissues. Exp Pathol 1982;21:79-88.

${ }^{13}$ Purnell DM, Heatfield BM, Trump BF. Immunocytochemical evaluation of human prostatic carcinomas for carcinoembryonic antigen, nonspecific cross-reacting antigen, $\beta$-chorionic gonadotrophin, and prostate-specific antigen. Cancer Res 1984; 44:285-92.

${ }^{14}$ Goldenberg DM, Sharkey RM, Primus FJ. Carcinoembryonic antigen in histopathology: immunoperoxidase staining of conventional tissue sections. J Natl Cancer Inst 1976;57:11-22.

is Goldenberg DM, Sharkey RM, Primus FJ. Immunocytochemical detection of carcinoembryonic antigen in conventional histopathology specimens. Cancer 1978;42:1546-53.

${ }^{10}$ Wahren B. Cellular content of carcinoembryonic antigen in urothelial carcinoma. Cancer 1978;42:1533-9.

17 Wiley EL, Mendelsohn G, Droller JM, Eggleston JC. Immunoperoxidase detection of carcinoembryonic antigen and blood group substances in papillary transitional cell carcinoma of the bladder. $J$ Urol 1982;128:276-80.

${ }^{18}$ Derechin M, Ostrowski W, Galka M. Acid phosphomonesterase of human prostate. Molecular weight, dissociation and chemical composition. Biochem Biophys Acta 1971;250: 143-54.

${ }^{19} \mathrm{Li}$ C-Y, Lam WKW, Yam LT. Immunohistochemical diagnosis of prostatic cancer with metastasis. Cancer 1980;46:706-12.

${ }^{20}$ Jobsis AC, de Vries GP, Meijer AEFH, Ploem JS. The immunohistochemical detection of prostatic acid phosphatase: its possiblities and limitations in tumour histochemistry. Histochem J 1981;13:961-73.

21 Yam LT, Janckila AJ, Lam WKW, Li C-Y. Immunohistochemistry of prostatic acid phosphatase. Prostate 1981;2:97-107.

22 Cohen C, Bentz MS, Budgeon LR. Prostatic acid phosphatase in carcinoid and islet cell tumours. Arch Pathol Lab Med 1983; 107: 277.

${ }^{23}$ Heyderman E. Tumour markers. In: Polak JM, Van Noorden S, eds Immunocytochemistry: practical applications in pathology and biology. Bristol: John Wright and Sons Ltd, 1983:274-94.

${ }^{24}$ Heyderman E. Immunoperoxidase technique in histopathology: applications, methods, and controls. J Clin Pathol 1979;32:971-8.

2s Nakane PK, Kawaio A. Peroxidase-labeled antibody: a new method of conjugation. $J$ Histochem Cytochem 1974;22: 1084-91.

${ }^{26}$ Heyderman E, Gibbons AR, Rosen SW. Immunoperoxidase localisation of human placental lactogen: a marker for the placental origin of the giant cells in "syncytial endometritis" of pregnancy. J Clin Pathol 1981;34:303-7.

${ }^{27}$ Wang MC, Valenzuelo L, Murphy GP, Chu TM. Purification of a human prostate specific antigen. Invest Urol 1979;17:159-63.

${ }^{2 R}$ Richardson TC, Heydermann E. Comparison between the enzymic and the immunological demonstration of prostatic acid phosphatase immobilized on agarose beads. Biochem Soc Trans 1984 (in press).

${ }^{24}$ Nadji M, Tabei Z, Castro A, Morales AR. Immunohistological demonstration of prostatic origin of malignant neoplasms. Lancet 1979; i: 671-2.

${ }^{30}$ Nadji M, Tabei SZ, Castro A, Ming Chu T, Morales AR. Prostatic original tumours. An immunohistochemical study. Am J Clin Pathol 1980; 73:735-9.

" Bentz MS, Cohen C, Demers LM, Budgeon LR. Immunohistochemical acid phosphatase level and tumour grade in prostatic carcinoma. Arch Pathol Lab Med 1982; 106:476-80. 
12 Vernon SE, Williams WD. Pre-treatment and post-treatment evaluation of prostatic adenocarcinoma for prostatic specific acid phosphatase and prostatic specific antigen by immunohistochemistry. J Urol 1983; 130:95-8.

"Shevchuk MM, Romas NA, Ng PY, Tannenbaum M, Olsson CA. Acid phosphatase localization in prostatic carcinoma. Cancer 1983:52: 1642-6.

is Isaacson P, Le Vann HP. The demonstration of carcinoembryonic antigen in colorectal carcinoma and colonic polyps using an immunoperoxidase technique. Cancer 1976;38: $1348-56$.

" Wiley EL. Mendelsohn G, Eggleston JC. Distribution of carcinoembryonic antigens and blood group substances in adenocarcinoma of the colon. Lab Invest 1981;44:507-13.

io Mach J-P. Putztaszeri G. Carcinoembryonic antigen (CEA):
Demonstration of a partial identity between CEA and a normal glycoprotein. Immunochemistry 1972;9: 1031-4.

${ }^{37}$ Von Kleist S, Chavanel G, Burtin B. Identification of a normal antigen that cross-reacts with the carcinoembryonic antigen. Proc Natl Acad Sci 1972;69:2492-4.

${ }^{3 *}$ Sloane JP, Ormerod MG, Neville AM. Potential pathological application of immunocytochemical methods to the detection of micrometastases. Cancer Res 1980;40:3079-82.

Requests for reprints to: Dr Eadie Heyderman, Department of Histopathology, St Thomas's Hospital, London SE1 7EH, England. 